\title{
Role of the CRISPR system in controlling gene transcription and monitoring cell fate (Review)
}

\author{
STELLA BALIOU $^{1}$, MARIA ADAMAKI ${ }^{1}$, ANTHONY M. KYRIAKOPOULOS ${ }^{2}$, DEMETRIOS A. SPANDIDOS ${ }^{3}$, \\ MICHALIS PANAYIOTIDIS ${ }^{4}$, IOANNIS CHRISTODOULOU ${ }^{1}$ and VASSILIS ZOUMPOURLIS ${ }^{1}$ \\ ${ }^{1}$ National Hellenic Research Foundation, 11635 Athens; ${ }^{2}$ Nasco AD Biotechnology Laboratory, 18536 Pireus; \\ ${ }^{3}$ Laboratory of Clinical Virology, Medical School, University of Crete, 71003 Heraklion, Greece; \\ ${ }^{4}$ Department of Applied Sciences, Northumbria University, Newcastle Upon Tyne NE1 8ST, UK
}

Received August 2, 2017; Accepted October 25, 2017

DOI: $10.3892 / \mathrm{mmr} .2017 .8099$

\begin{abstract}
Even though the accrual of transcripts is implicated in distinct disease states, our knowledge regarding their functional role remains obscure. The CRISPR system has surged at the forefront of genome engineering tools in the field of RNA modulation. In the present review, we discuss some exciting applications of the CRISPR system, including the manipulation of RNA sequences, the visualization of chromosomal loci in living cells and the modulation of transcription. The CRISPR system has been documented to be very reliable and specific in altering gene expression, via leveraging inactive catalytically dead CRISPR-associated protein 9 (Cas9). In the present review, the CRISPR system is presented as an eminent tool for the meticulous analysis of gene regulation, loci mapping and complex pathways.
\end{abstract}

\section{Contents}

1. Mechanisms of action of the CRISPR system

2. Controlling gene expression through the CRISPR system

3. Approaches for spatiotemporal control of gene expression

4. Progress made in elucidating the role of non-coding RNAs using the CRISPR system

5. Exploiting the CRISPR/Cas approach in the field of epigenetics

6. Implementation of the CRISPR system in the field of imaging

7. Utilizing the CRISPR system in monitoring cell fate

8. Conclusions

Correspondence to: Dr Vassilis Zoumpourlis, National Hellenic Research Foundation, 48 Vasileos Konstantinou Avenue, 11635 Athens, Greece

E-mail:vzub@eie.gr

Key words: gene regulation, CRISPR, inactive CRISPR-associated protein 9, imaging, epigenetics, non-coding RNAs

\section{Mechanisms of action of the CRISPR system}

In general, the main function of the CRISPR system is mediated by the CRISPR-associated protein (Cas) nuclease, which interacts with DNA and generates double-strand breaks in DNA sequence, matching the broken genomic region with a single guide RNA (sgRNA). The sgRNA is a chimeric RNA which consists of programmable CRISPR RNA and a trans-activating RNA (tracrRNA) (1). More specifically, the CRISPR-Cas system includes a cluster of proteins, categorized into Class 1 (Types I, III and IV) and Class 2 (Types II, V and VI) (2), all of which constitute specific RNA-guided DNA endonuclease (Cas) proteins (1-4). A distinct characteristic of Cas proteins is that they are driven by RNAs, and not by other proteins, in recognizing the desired DNA sequence. The CRISPR-Cas subtype that is usually selected is Class 2, which generally exploits Cas 9 nuclease $(1,3,4)$. As regards the RNA-mediated action of the CRISPR system, it has been reported that the $100 \mathrm{bp}$ sgRNA forms complementary bonds with the target DNA sequence of 17-20 nucleotides, via WatsonCrick base-pairing and the tracrRNA is the component which Cas9 nuclease binds to. Specifically, the sgRNA recognizes the target sequence located upstream of a triplicate sequence named the protospacer adjacent motif (PAM), given that the PAM motif recruits Cas9 nuclease at the site of DNA cleavage (5) (Fig. 1). Of note, the PAM sequence plays a determinant role in recognizing the correct DNA sequence and preventing the direction of RNA to self-targets and non-specific sequences (6). In the case of Cas9 that is derived from Streptococcus pyogenes, the motif of the PAM sequence may be composed of any base, followed by two additional guanine bases (7). In addition, the superiority of Cas9 nuclease has been demonstrated not only against other nucleases, but also against the silencing mechanism, known as RNA interference (RNAi), that has been used extensively for the disruption of gene expression. Another important aspect is that the CRISPR genome editing tool acts in the nucleus, whereas the RNAi system exerts its action in the cytoplasm. RNAi seems to have prevalent unpredictable off-target effects (8) due to the extensive modulation of microRNAs (miRNAs or miRs) (9) or partial silencing (10). Compared to RNAi techniques, the CRISPR system functions at the DNA level, resulting in the permanent inactivation 

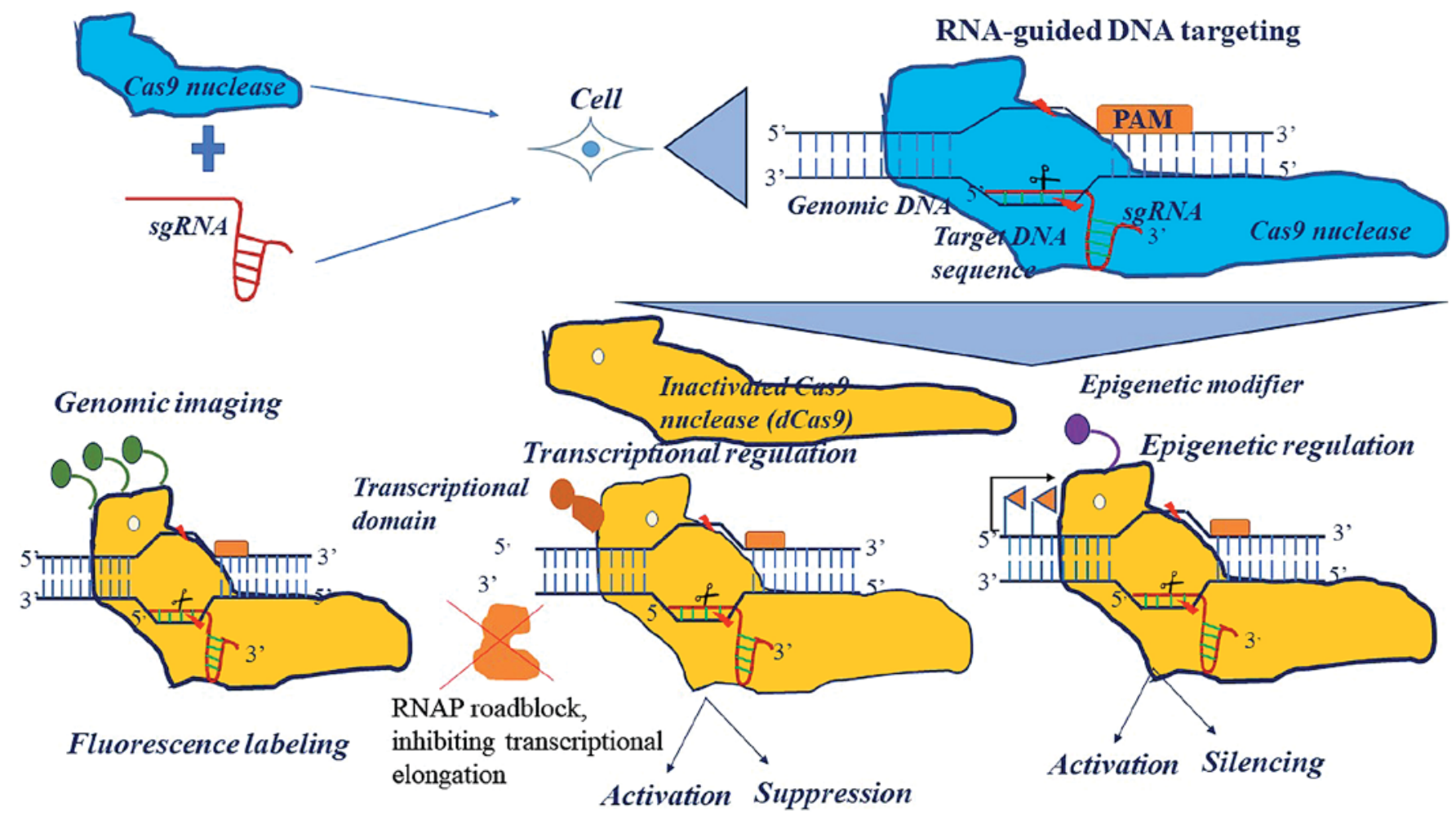

Figure 1. The CRISPR platform for gene editing, genomic regulation and imaging.

of a given gene, with fewer off-target effects. On the other hand, the main disadvantage of the CRISPR system is its low efficiency in some cases, as its effects are undetectable in a cell population, thus prompting researchers to optimize the system for implementation in the clinical setting.

As regards the challenges associated with the CRISPR technique, the method has been referred to sustain its activity even if the sgRNA and the target sequence do not form complementary bonds in 3 sequential bases at the $3^{\prime}$ terminal end of sgRNA (11). It has also been mentioned that the scale of side-effects begins at 10 and exacerbates to 1,000 . However, the phenomenon can be partially rescued with the careful design of guide RNA (gRNA) (12); characteristically, the specificity of the CRISPR system can be accelerated by reducing the sequence of gRNA to 17-18 nucleotides (13).

\section{Controlling gene expression through the CRISPR system}

Advances in the CRISPR engineering tool have led to the creation of inactivated (catalytically dead) Cas9 (dCas9). Modified Cas9 involves the following: Catalytic substitution (D10A) in the RuvC domain, the abolishment of endonuclease activity and catalytic replacement (H847A) in the HNH domain.

dCas9 bound to DNA elements alone may inhibit the transcription either at the initiation or the elongation level. Specifically, it has been demonstrated that dCas9 can impair transcription, excluding the potential of crosstalk between cis-acting motifs of any promoter with trans-acting transcription factors during transcriptional initiation (14). In the process of transcriptional elongation, the DNA is not cleaved due to the lack of dCas9 endonuclease activity and the RNA polymerase is sterically prevented, causing transcriptional stalling in a strand-specific manner (14). It is evident that the association between the sgRNA-dCas9 complex and RNAP is responsible for transcriptional elongation.

Apart from simply blocking the transcription of target genes, it may be possible to couple the $\mathrm{dCas} 9$ protein with numerous regulatory domains, modulate different biological processes and generate functional outcomes. Importantly, the inactivated domain of Cas 9 can be combined with activator or repressor domains, thus controlling gene expression. The inactivated domain of Cas9 can be fused to epigenetic modifiers, thus paving the way in the control of gene expression and epigenetic modifications in a fast and reliable manner (15) (Fig. 1).

In the case of dCas9, the DNA is not cleaved due to the lack of Cas9 endonuclease activity. The dCas9 can be fused to transcription factor domains or epigenetic modifiers or fluorescent molecules, thus determining the control of gene expression or imaging, respectively.

Overall, the control of gene expression can be mediated in a spatial-temporal manner, via the binding of dCas9 with a repressor domain, such as Kruppel-associated box (KRAB) (16) or an activator domain, such as VP16 and VP64 (17), thereby inhibiting or triggering gene expression, respectively. On the one hand, the interruption of transcription in target genes can be culminated, via a combination of dCas9 with transcriptional repressor domains (KRAB or SID) and epigenetic modifiers that are responsible for epigenetic silencing $(17,18)$. In CRISPR interference (CRISPRi), the transcriptional silencing is very high at both the prokaryotic and the eukaryotic level (14). Of note, CRISPRi can repress the transcription of an endogenous gene or of multiple genes independently in bacterial and mammalian cells, as illustrated by Mandegar et al by eliminating the factors, octamer-binding transcription factor 4 (OCT4), 
NANOG and sex determining region Y)-box 2 (SOX2), the kinases, Rho-associated, coiled-coil-containing protein kinase 1 (ROCK1) and glycogen synthase kinase 3 (GSK3)- $\beta$, a cardiac mesoderm transcription factor, and cardiac diseaseassociated genes [BCL2-associated athanogene 3 (BAG3), myosin binding protein $\mathrm{C}$ (MYBPC) 3 and human ethera-go-go-related gene (hERG)] in induced pluripotent stem cells (iPSCs) (19). The repressor activity of CRISPRi has only been validated using quantitative fluorescence assays and native elongating transcript sequencing (20). On the other hand, Cas9 nuclease can boost gene transcription via coupling with VP16/VP64 or p65 activation domains. It is noteworthy that the level of transcriptional upregulation depends on the presence of single $(18,21-23)$ or multiple sgRNAs which have a cumulative effect on transcription (21-23). In order to augment the activation signal, researchers have used a SunTag array, in which dCas9 is fused to a protein platform loaded with 24 epitopes, each of which is recognized by a single chain variable fragment (scFv) of antibody, which is in turn linked to activator effector domains like VP64 (24). For example, it has been reported that CXCR4 expression is more prominently augmented (50 fold) with the SunTag array, as compared to a 2-fold increase that is mediated by the action of dCas9-VP64 (25). In the same context, the significant upregulation of gene expression can be mediated by 'in trans' interactions through synergistic activation mediator (SAM). The SAM approach is reportedly based on bringing activators (e.g., p65-HSF1) fused with MS2 bacteriophage coat protein and Cas9 loaded with VP64 to interact with sgRNAs containing MS2 target sites (26). Furthermore, a method of enhancing the activation of gene expression though dCas9-VPR (VP64, p65 and Rat) has been presented, which specifically exploits the synergistic power of distinct activating factors 'in cis' (27).

Based on the ability of RNA to function as a scaffold, Zalatan et al (28) were inspired to design a CRISPR sgRNA sequence which was fused to single RNA hairpin domains via a linker of 2 bases, using viral sequences that recruit the assembly of RNA-binding factors. In turn, the RNA-binding factors orchestrated the landscape for transcriptional activation or suppression of target genes, via interaction with the appropriate transcription factors. As a result, the expression of multiple genes was controlled simultaneously, either by activation or suppression, whereas at the same time this unique system allowed for large scale screening of pairwise combinations of genes, many of which constituted important regulators of tumorigenesis (28).

In all cases, there are certain factors that influence the outcome of upregulated or attenuated gene expression. The orientation of $\mathrm{sgRNAs}$ relative to the transcriptional start site (TSS) of genes and the selection between amino terminal or carboxyterminal fusion of dCas 9 with effector domains may be of fundamental importance in determining the end transcriptional result. For example, it has been noted that the well-established VPR system of transcriptional activation can also be exploited for the suppression of endogenous genes when gRNAs are orientated downstream of the TSS (29).

Despite the considerable number of studies on tumorigenesis, there is a certain paucity of data pertinent to various regulatory elements, including enhancers. For example, the functional involvement of enhancers in tumorigenesis has been validated through their capacity to orchestrate gene expression profiles (30). Classical research methods, such as high-throughput reporter assays, have been employed to address enhancer properties (31); however, inadequate results have been produced. Nowadays, the revolutionary CRISPR approach provides us with the opportunity to monitor the distribution of regulatory elements and determine their functional properties. Impressively, Xie et al (32) devised a conceptual framework (Mosaic-seq), which utilizes a CRISPR barcoding approach in combination with single-cell RNA sequencing by Drop-seq, to quantitatively evaluate enhancer function and identify the elements of super enhancers (32). Specifically, K562 cells infected with dCas9 fused to the KRAB domain, resulting in the repression of gene expression at potential enhancers; subsequently, the cells were infected with a labeled sgRNA library against possible enhancers and assorted following the application of antibiotic tests for RNA sequencing (Drop-seq). Mosaic-seq has offered insight into enhancer penetrance, endogenous activity and a deeper understanding of the hierarchy of the constituents that comprise super enhancers in a genome-wide, single-cell and unbiased manner (32).

For the study of promoter sequences, Chiba et al used the CRISPR system to trigger cancer-related TERT promoter deletions, in order to delineate the consequences in neoplastic as well as normal cells, such as human embryonic stem cells (33). In other words, the particular genome engineering tool allows researchers to identify the cause of aberrantly long telomeres in tumorigenesis.

\section{Approaches for spatiotemporal control of gene expression}

The CRISPR system can be further evaluated to function in a spatial-temporal manner and many scientific advances have been made in this direction. One method that has garnered significant attraction is gene manipulation according to induction by light. For example, the heterodimerization of CRY2 and CIB1 proteins has been observed in response to $450 \mathrm{~nm}$ light, allowing the assembly of VP64 to dCas9 (34). Another method supports that Cas9 nuclease is uncoupled to its gRNA in the absence of light. The researchers have substantiated that a lysine residue (K866) is responsible for the activation of Cas9 nuclease, given that conformational changes are performed upon binding of sgRNA and UV illumination (35). Another method has employed the segregation of Cas9 nuclease and its conjunction with photoinducible partners. The action of the CRISPR system is performed only after irradiation with blue light in which the distinct segments of Cas9 nuclease are assembled in a complex (36). Last but not least, the inducible effect of the CRISPR system on gene expression can be mediated by a chemical stimulus (doxycycline, rapamycin and 4-hydroxytamoxifen) (37,38).

\section{Progress made in elucidating the role of non-coding RNAs using the CRISPR system}

Despite the reported advances in generating schemes for the insertion or elimination of genes, there appears to be a lack of strategies that can be applied at the RNA level. The CRISPR approach is not restricted to the modification of the coding 
compartment of the cell, but it also applies to the non-coding compartment, which usually has a significant regulatory functions.

The advantage of CRISPRi over RNAi is that the former is an exogenous system that does not intervene with endogenous processes and facilitates the study of long-noncoding RNAs (lncRNAs), as well as miRNAs. The CRISPRi system is based on the action of catalytically dCas9 which does not exert endonuclease activity $(14,17)$. Importantly, dCas9 abrogates the transcriptional machinery, thus hindering gene expression by exploiting the sgRNA sequence only, and maps the presence of cis regulatory element binding in the lactose regulatory network (19). For the CRISPRi system to function properly, sgRNAs should be designed such that the distance between the TSS and the target should be approximately 300 bp (39), so as to avoid mismatches and side-effects.

In modulating gene expression, lncRNAs have been shown to play a fundamental role in both normal developmental processes and in abnormal cellular states such as cancer, thus highlighting the need for further investigation (40). IncRNAs have been reported to be modified by the CRISPR approach only (41). The CRISPR system acts by either disrupting the target lncRNA or by inserting RNA-destabilizing elements or by introducing a poly-adenylation stop signal, thus leading to gene replacement or promoter disruption (40). Several research groups have employed CRISPR strategies to disrupt the function of lncRNAs in mouse models. The ablation of the Malat1 lncRNA constitutes an indicative example of the above strategy (42). An alternative strategy supports the replacement of an lncRNA sequence with a marker sequence (such as LacZ), thus allowing the monitoring of the reporter gene expression as driven by the promoter of the lncRNA (43). However, that method was relatively costly and tested on mice that were specifically deficient for 18 lncRNAs (43). Notably, it was previously demonstrated that the HOXA gene cluster can be regulated by the IncRNA transcript known as Haunt. Using the CRISPR system, it was found that Haunt lncRNA functioned as a roadblock, impairing HOXA gene expression when it was assigned as aberrant (44). Therefore, the CRISPR genome engineering tool appears to be the first method for the identifaction of the molecular mechanisms mediated by lncRNAs in various biological processes.

Nonetheless, the implementation of the CRISPR method requires specific information regarding the traits of lncRNAs, such as their effect on the 'cis' or 'trans' expression of genes or on the expression of multiple genes. For example, only approximately $40 \%$ of assigned lncRNAs can be subjected to the CRISPR screen, where gene sequences near the IncRNAs may be influenced by the action of Cas9 nuclease and the danger of no specificity has not been circumvented.

As regards the study of miRNAs, Xiao et al were the first group that used the CRISPR method to eliminate large genomic regions of $1 \mathrm{Mb}$ in size in zebrafish, thereby suppressing the action of miRNAs that constitute 1 or $3 \%$ of the zebrafish genome (dre-mir-126a or miRNA cluster Chr. 9) (45). In particular, the CRISPR method was used to suppress the following: miR-137 in ovarian cancer (46), the UCA1 lncRNA in bladder cancer (47), as well as the Hotair (HOX transcript antisense RNA) (48) and BC200 lncRNAs in breast cancer (49). Furthermore, the inverse association between miR-24-3p and
E3 ubiquitin ligase and the TRIM11 oncogene was previously investigated by the CRISPR method, through which the attenuation of colorectal tumor progression was facilitated and a novel therapeutic target in colorectal cancer was highlighted (50).

Last but not least, the Molecular Chipper approach was previously used to investigate the functional regulatory elements involved in the biogenesis of lncRNAs, highlighting important cis-domains for miR-142 biogenesis (51). In the same direction, Shechner et al demonstrated an innovative CRISPR-Display method in studying the functional significance of regulatory molecules such as lncRNAs, miRNAs, circular RNAs, piwi-interacting RNAs and many others types of RNA (52). The pioneering CRISPR-Display platform was designed to shed further light on the molecular mechanisms exerted by lncRNAs on a spectrum of biological processes, such as epigenetic modifications, transcriptional regulation and chromatin remodeling. The CRISP-Display platform has allowed researchers to evaluate the functionality of RNA molecules, either based on their transcriptional profile or on topological changes and alterations in the tridimensional landscape (52). A characteristic example of exploiting accessory RNA domains was demonstrated by Shechner et al, who conjugated the RepA domain of Xist with gRNA. Therefore, the repressive action of Xist, caused by its association with the RepA domain, was unveiled. The CRISPR-Display platform also functions in a flexible and multiplex manner, as it facilitates fusing the gRNA to a lncRNA and monitoring of the transcription of a reported gene with concomitant imaging of another DNA locus, thus allowing for simultaneous analysis of several targets based on the available RNA motifs (53).

The main characteristic of the CRISP-Display platform is the delivery of dCas9 to predetermined DNA loci according to the specificity of gRNA and the dCas9 fusion with any of transcriptional effector domains, including VP4 (52). As regards the RNA cargos used by Cas9, it was indicated that effector domains can be linked to dCas9 nuclease either directly (known as 'direct activation) or indirectly, using aptamers which can function as 'accessory RNA domains' of $4.8 \mathrm{~kb}$ in size, with the potential of binding the suitable transcriptional domain (knownas 'bridged' activation) without sterical barriers, given that the RNA domains can be positioned at the $5^{\prime}$ or the $3^{\prime}$ end of gRNA. The only restraint in implementing the CRISP-Display approach in all the non-coding RNAs was the restricted panel of RNA domains that can be appended to sgRNAs (52).

\section{Exploiting the CRISPR/Cas approach in the field of epigenetics}

The etiology of several diseases may be linked to epigenetic alterations, which may have a significant impact on the transcriptional level or on DNA methylation patterns or histone modifications, thus altering diverse biological functions such as transcription. It has been demonstrated that mutations in inactive nucleases cause them to be fused with epigenetic modifiers and the transcriptional landscape is reorganized. Stable transcriptional suppression has been achieved through fusion of dCas9 with a repression domain such as KRAB and DNA methylatransferase (53). Specifically, Cas9 nuclease has been successfully fused with the Dnmt3 


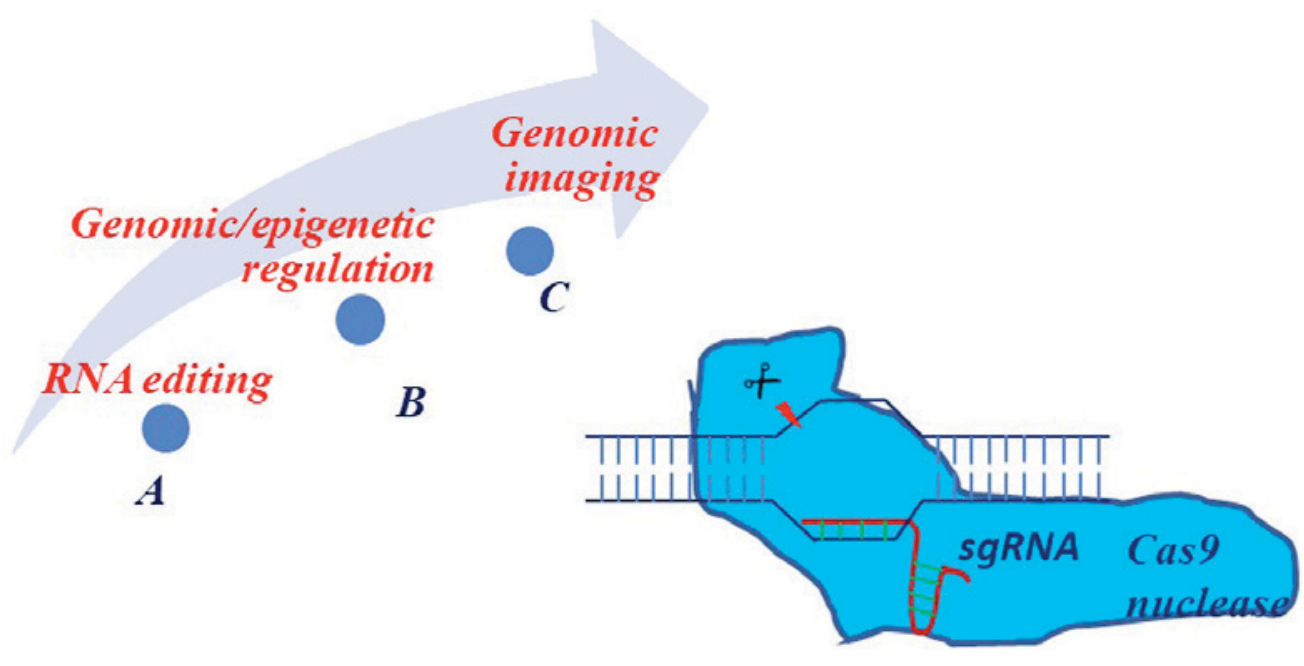

Figure 2. (A) RNA editing at non-coding RNAs, such as lncRNAs, miRNAs. (B) Modulation of gene expression in a spatial-temporal manner and epigenetic regulation using dCas9 coupled to epigenetic modifiers. (C) Genomic imaging using dCas9 fused to a fluorescent marker. lncRNAs, long-noncoding RNAs; miRNAs, micro-RNAs; dCas9, inactivated Cas9.

DNA methyltransferases (54) and with the G9a or SUV39H1 methyltransferase to mediate epigenetic silencing. Notably, the synergistic effect of the dCas9 repressor domain fused to DNA methyltransferasesDNMT3A and DNMT3L appears to be more effective than using the dCas9-repressor domain alone (55).

By contrast, the fusion of dCas9 to other epigenetic modulators, such as TET1 demethylase (56), LSD1 histone demethylase or the p300 histone acetyltransferase (57) seems to upregulate endogenous genes. Nonetheless, experimental methods need to be further investigated in order to elucidate the crosstalk between epigenetic modifiers and endogenous proteins, as they often cause false phenotypes (58).

\section{Implementation of the CRISPR system in the field of imaging}

The organization of chromatin in the nucleus is usually addressed as a critical issue. Identifying the principles behind chromatin orientation can help in the understanding of how the spatial organization of chromatin can affect gene expression. For this purpose, FISH technologies that utilize the denaturation of double-strand DNA, heating/formamide and the attachment of fluorescent probes to denatured DNA have been extensively used. The main drawbacks of the available techniques are disruption of the genomic structure, restricted resolution and the prohibitive expense of oligo probes (59).

Previous data have demonstrated an impressive technique to monitor not only the repetitive elements in telomeres, but also gene sequences in live cells, circumventing the classical problems of fixation and DNA denaturation that emerge during FISH technologies or artificial insertions of $\mathrm{LacO}$ or TetO cassettes (60). Specifically, Chen et al modified a form of nuclease Cas9 [coupled with a fluorescent molecule, such as enhanced green fluorescent protein (EGFP)] and optimized small guide RNA, thus managing to monitor the route of desired genomic sequences in live cells and track the dynamic nature of chromatin (61). In the meantime, Cas9 nuclease appeared to be a more powerful as compared to the transcription activator-like effector nuclease that had been usually used for detecting telomere and satellite DNA (62), as Cas9 nuclease has the capacity to label multiple genomic sequences in live cells. Consequently, imaging using Cas9 nuclease proved to be a significant advancement, not only in terms of relating chromatin state with gene function, but also in understanding the aneuploidy that is usually seen in cancer cells (63). In addition, CRISPR imaging appears to be very effective in studying interactions among genomic elements as compared to other techniques (chromatin conformation capture-3C and derivatives such as Hi-C) (64). Importantly, Cas9 nuclease was recently modulated to monitor RNA in live cells instead of RNA FISH/MeCP2, thus facilitating visualization of the endogenous RNA distribution in live cells without exogenous tags, and even their accumulation in stress granules of live cells (65). A particularly innovative technology was devised for the detection of genes within undisturbed nuclei of fixed cells and tissues; Cas9 and CRISPR repeats (clustered regularly interspaced short palindromic repeats) were combined with an RNA sequence to serve as probes in identifying the genes of interest at the genome level. That approach not only preserved the spatial relationships of the genetic elements, which appear very important in understanding gene expression, but the process was overall remarkably quick (15 min), convenient and amenable to diagnostic tests. Previous approaches have used fluorescent DNA probes to hybridize to genes of interest, which often require heat treatment and disruptive chemicals that distort the natural organization of the nucleus.

Furthermore, the combination of inactivated $\mathrm{Ca} 9$ and EGFP has proven to be very sufficient in monitoring the localization of any genomic sequence, apart from the telomeres in which it was first applied (61). In this direction, another advantageous method for enriching the signal in live cell imaging is to use the dCas9-SunTag system, in which fluorescent molecules are fused to individual $\mathrm{scFv}$ which are located on a protein epitope tail fused to dCas9 (24). In addition, phototoxicity has been minimized by reducing excitation laser power and subsequently by amplifying the signal at a specific target (24). If one 
considers the scaffold properties of sgRNA (50), random RNA aptamers could be exploited for the assembly of fluorescent molecules (66), instead of using epitopes.

\section{Utilizing the CRISPR system in monitoring cell fate}

Despite being essential tools in the identification of tumorigenic genes, pooled genetic screens cannot be used to analyze neoplasm cellular complexity and clonality, as the results derived from screens can lead to false-negative and -positive conclusions (67).

Based on the notion that cancer is a stochastic procedure in which cancer cells adapt to the environment by acquiring 'de novo' mutations, researchers have revealed a new barcoding system that enables monitoring the fate of cancer subpopulations. Notably, the tumor immune escape is attributed to complex tumor heterogeneity, possibly pertaining to the origin of cancer cells. In the CRSIPR-barcoding system, a homology directed repair mechanism has been used to specifically introduce mutations in cancer cells, along with silent mutations as a reference, thus recapitulating the clonal history of cells. It has been demonstrated that CRISPR-barcoding is multiplex, allowing the remodeling of many mutations in cancer cell populations and leading to a more comprehensive understanding of cellular networks. The effects of distinct drug combinations (gefitinib-WZ4002, ALK inhibitor TAE684-WZ4002, gefitinib-trametinib) in prostate cancer cells (PC9 cells) harboring either KRAS G12D mutation or EGFR T790M mutation or EML4-ALK rearrangement have been deciphered. In addition, the effects caused by inactivation of the p53 gene (TP53 R273H) in breast cancer cells (MCF7 cells) and colon cancer cells (HCT-116 cells) have been identified, whereas the expression of adenomatous polyposis coli gene (APC) in colon cancer cells (DLD-1 cells) has been abrogated. Furthermore, CRISPR-barcoding has been employed to repair the ALK-F1174L activating mutation in Kelly neuroblastoma cells, resulting in correction of the mutation or in generating truncated protein (ALK-STOP barcode). Overall, this new CRISPR-barcoding tool has facilitated the monitoring and study of cell fate, both in response to therapy and in the absence of therapy. The new strategy may be used for the identification of any type of genetic modification, is compatible with many cancer types and functional assays, but above all it does not cause side effects owing to silent mutations, thus paving the way for the development of new therapeutic protocols (68).

\section{Conclusions}

The CRISPR system has been employed for the enhancement or attenuation of gene expression in a more reliable manner as compared to previous genome engineering techniques. The innovative use of the CRISPR system has been implemented to modulate gene expression through the alteration of epigenetic modifiers or crucial noncoding RNAs. Importantly, the CRISPR system is of utmost importance in delineating the role of non-coding RNAs (miRNAs, lncRNAs) which are often implicated in tumor progression and heterogeneity (Fig. 2). Finally, the CRISPR system constitutes a flexible platform that has been used for monitoring the localization of endogenous genes or cell fate in general.

\section{References}

1. Wright AV, Nuñez JK and Doudna JA: Biology and applications of CRISPR systems: Harnessing nature's toolbox for genome engineering. Cell 164: 29-44, 2016.

2. Wiedenheft B, Sternberg SH and Doudna JA: RNA-guided genetic silencing systems in bacteria and archaea. Nature 482: 331-338, 2012.

3. van der Oost J, Westra ER, Jackson RN and Wiedenheft B: Unravelling the structural and mechanistic basis of CRISPR-cas systems. Nat Rev Microbiol 12: 479-492, 2014.

4. Mougiakos I, Bosma EF, de Vos WM, van Kranenburg R and van der Oost J: Next generation prokaryotic engineering: The CRISPR-cas toolkit. Trends Biotechnol 34: 575-587, 2016.

5. Bolotin A, Quinquis B, Sorokin A and Ehrlich SD: Clustered regularly interspaced short palindrome repeats (CRISPRs) have spacers of extrachromosomal origin. Microbiology 151: 2551-2561, 2005.

6. Westra ER, Semenova E, Datsenko KA, Jackson RN, Wiedenheft B, Severinov K and Brouns SJ: Type I-E CRISPR-cas systems discriminate target from non-target DNA through base pairing-independent PAM recognition. PLoS Genet 9: e1003742, 2013.

7. Mojica FJ, Díez-Villaseñor C, García-Martínez J and Almendros C: Short motif sequences determine the targets of the prokaryotic CRISPR defence system. Microbiology 155: 733-740, 2009.

8. Jackson AL, Bartz SR, Schelter J, Kobayashi SV, Burchard J, Mao M, Li B, Cavet G and Linsley PS: Expression profiling reveals off-target gene regulation by RNAi. Nat Biotechnol 21: 635-637, 2003.

9. Marine S, Bahl A, Ferrer M and Buehler E: Common seed analysis to identify off-target effects in siRNA screens. J Biomol Screen 17: 370-378, 2012.

10. Moore JD: The impact of CRISPR-Cas9 on target identification and validation. Drug Discov Today 20: 450-457, 2015.

11. Ran FA, Hsu PD, Wright J, Agarwala V, Scott DA and Zhang F: Genome engineering using the CRISPR-Cas9 system. Nat Protoc 8: 2281-2308, 2013.

12. Kuscu C, Arslan S, Singh R, Thorpe J and Adli M: Genome-wide analysis reveals characteristics of off-target sites bound by the Cas9 endonuclease. Nat Biotechnol 32: 677-683, 2014.

13. Fu Y, Sander JD, Reyon D, Cascio VM and Joung JK: Improving CRISPR-cas nuclease specificity using truncated guide RNAs. Nat Biotechnol 32: 279-284, 2014.

14. Qi LS, Larson MH, Gilbert LA, Doudna JA, Weissman JS, Arkin AP and Lim WA: Repurposing CRISPR as an RNA-guided platform for sequence-specific control of gene expression. Cell 152: 1173-1183, 2013.

15. Boettcher M and McManus MT: Choosing the right tool for the job: RNAi, TALEN, or CRISPR. Mol Cell 58: 575-585, 2015.

16. Lawhorn IE, Ferreira JP and Wang CL: Evaluation of sgRNA target sites for CRISPR-mediated repression of TP53. PLoS One 9: e113232, 2014.

17. Gilbert LA, Larson MH, Morsut L, Liu Z, Brar GA, Torres SE, Stern-Ginossar N, Brandman O, Whitehead EH, Doudna JA, et al: CRISPR-mediated modular RNA-guided regulation of transcription in eukaryotes. Cell 154: 442-451, 2013.

18. Konermann S, Brigham MD, Trevino A, Hsu PD, Heidenreich M, Cong L, Platt RJ, Scott DA, Church GM and Zhang F: Optical control of mammalian endogenous transcription and epigenetic states. Nature 500: 472-476, 2013.

19. Mandegar MA, Huebsch N, Frolov EB, Shin E, Truong A Olvera MP, Chan AH, Miyaoka Y, Holmes K, Spencer CI, et al: CRISPR interference efficiently induces specific and reversible gene silencing in human iPSCs. Cell Stem Cell 18: 541-553, 2016.

20. Larson MH, Gilbert LA, Wang X, Lim WA, Weissman JS and Qi LS: CRISPR interference (CRISPRi) for sequence-specific control of gene expression. Nat Protoc 8: 2180-2196, 2013.

21. Maeder ML, Linder SJ, Cascio VM, Fu Y, Ho QH and Joung JK: CRISPR RNA-guided activation of endogenous human genes. Nat Methods 10: 977-979, 2013.

22. Mali P, Aach J, Stranges PB, Esvelt KM, Moosburner M, Kosuri S, Yang L and Church GM: CAS9 transcriptional activators for target specificity screening and paired nickases for cooperative genome engineering. Nat Biotechnol 31: 833-838, 2013.

23. Perez-Pinera P, Kocak DD, Vockley CM, Adler AF, Kabadi AM, Polstein LR, Thakore PI, Glass KA, Ousterout DG,Leong KW, et al: RNA-guided gene activation by CRISPR-Cas9-based transcription factors. Nat Methods 10: 973-976, 2013. 
24. Tanenbaum ME, Gilbert LA, Qi LS, Weissman JS and Vale RD: A protein-tagging system for signal amplification in gene expression and fluorescence imaging. Cell 159: 635-646, 2014.

25. La Russa MF and Qi LS: The new state of the art: Cas9 for gene activation and repression. Mol Cell Biol 35: 3800-3809, 2015.

26. Konermann S, Brigham MD, Trevino AE, Joung J, Abudayyeh OO, Barcena C, Hsu PD, Habib N, Gootenberg JS, Nishimasu H, et al: Genome-scale transcriptional activation by an engineered CRISPR-Cas9 complex. Nature 517: 583-588, 2015.

27. Chavez A, Scheiman J, Vora S, Pruitt BW, Tuttle M, P R Iyer E, Lin S, Kiani S, Guzman CD, Wiegand DJ, et al: Highly efficient Cas9-mediated transcriptional programming. Nat Methods 12: 326-328, 2015.

28. Zalatan JG, Lee ME, Almeida R, Gilbert LA, Whitehead EH, La Russa M, Tsai JC, Weissman JS, Dueber JE, Qi LS and Lim WA: Engineering complex synthetic transcriptional programs with CRISPR RNA scaffolds. Cell 160: 339-350, 2015

29. Braun CJ, Bruno PM, Horlbeck MA, Gilbert LA, Weissman JS and Hemann MT: Versatile in vivo regulation of tumor phenotypes by dCas9-mediated transcriptional perturbation. Proc Natl Acad Sci USA 113: E3892-E3900, 2016.

30. Mansour MR, Abraham BJ, Anders L, Berezovskaya A, Gutierrez A, Durbin AD, Etchin J, Lawton L, Sallan SE, Silverman LB, et al: Oncogene regulation. An oncogenic super-enhancer formed through somatic mutation of a noncoding intergenic element. Science 346: 1373-1377, 2014.

31. Inoue F, Kircher M, Martin B, Cooper GM, Witten DM, McManus MT, Ahituv N and Shendure J: A systematic comparison reveals substantial differences in chromosomal versus episomal encoding of enhancer activity. Genome Res 27: 38-52, 2017

32. Xie S, Duan J, Li B, Zhou P and Hon GC: Multiplexed engineering and analysis of combinatorial enhancer activity in single cells. Molecular cell 66: 285-99, 2017

33. Chiba K, Johnson JZ, Vogan JM, Wagner T, Boyle JM and Hockemeyer D: Cancer-associated TERT promoter mutations abrogate telomerase silencing. eLife 4: 4, 2015.

34. Polstein LR and Gersbach CA: A light-inducible CRISPR-Cas9 system for control of endogenous gene activation. Nat Chem Biol 11: 198-200, 2015

35. Hemphill J, Borchardt EK, Brown K, Asokan A and Deiters A Optical Control of CRISPR/Cas9 gene editing. J Am Chem Soc 137: 5642-5645, 2015.

36. Nihongaki $Y$, Kawano $F$, Nakajima $T$ and Sato $M$ : Photoactivatable CRISPR-Cas9 for optogenetic genome editing. Nat Biotechnol 33: 755-760, 2015.

37. Liu KI, Ramli MN, Woo CW, Wang Y,Zhao T, Zhang X, Yim GR, Chong BY, Gowher A, Chua MZ, et al: A chemical-inducible CRISPR-Cas9 system for rapid control of genome editing. Nat Chem Biol 12: 980-987, 2016

38. Zetsche B, Volz SE and Zhang F: A split-Cas9 architecture for inducible genome editing and transcription modulation. Nat Biotechnol 33: 139-142, 2015.

39. Gilbert LA, Horlbeck MA, Adamson B, Villalta JE, Chen Y, Whitehead EH, Guimaraes C, Panning B, Ploegh HL, Bassik MC, et al: Genome-scale CRISPR-mediated control of gene repression and activation. Cell 159: 647-661, 2014.

40. Haemmerle $M$ and Gutschner T: Long non-coding RNAs in cancer and development: Where do we go from here? Int J Mol Sci 16: 1395-1405, 2015

41. Hale CR, Zhao P, Olson S, Duff MO, Graveley BR, Wells L, Terns RM and Terns MP: RNA-guided RNA cleavage by a CRISPR RNA-Cas protein complex. Cell 139: 945-956, 2009.

42. Gutschner T: Silencing long noncoding RNAs with genome-editing tools. Methods Mol Biol 1239: 241-250, 2015.

43. Sauvageau M, Goff LA, Lodato S, Bonev B, Groff AF, Gerhardinger C, Sanchez-Gomez DB, Hacisuleyman E, Li E, Spence M, et al: Multiple knockout mouse models reveal lincRNAs are required for life and brain development. eLife 2: e01749, 2013

44. Yin Y, Yan P, Lu J, Song G, Zhu Y, Li Z, Zhao Y, Shen B, Huang $\mathrm{X}$, Zhu H, et al: Opposing roles for the lncRNA haun and its genomic locus in regulating HOXA gene activation during embryonic stem cell differentiation. Cell Stem Cell 16 504-516, 2015

45. Xiao A, Wang Z, Hu Y, Wu Y, Luo Z, Yang Z, Zu Y, Li W, Huang $\mathrm{P}$, Tong $\mathrm{X}$, et al: Chromosomal deletions and inversions mediated by TALENs and CRISPR/Cas in zebrafish. Nucleic Acids Res 41: e141, 2013.

46. Li X, Chen W, Zeng W, Wan C, Duan S and Jiang S: microRNA-137 promotes apoptosis in ovarian cancer cells via the regulation of XIAP. Br J Cancer 116: 66-76, 2017.
47. Zhen S, Hua L, Liu YH, Sun XM, Jiang MM, Chen W, Zhao L and $\mathrm{Li} \mathrm{X}$ : Inhibition of long non-coding RNA UCA 1 by CRISPR/Cas9 attenuated malignant phenotypes of bladder cancer. Oncotarget 8: 9634-9646, 2017.

48. Gupta RA, Shah N, Wang KC, Kim J, Horlings HM, Wong DJ, Tsai MC, Hung T, Argani P, Rinn JL, et al: Long non-coding RNA HOTAIR reprograms chromatin state to promote cancer metastasis. Nature 464: 1071-1076, 2010.

49. Singh R, Gupta SC, Peng WX, Zhou N, Pochampally R, Atfi A, Watabe K, Lu Z and Mo YY: Regulation of alternative splicing of Bcl-x by BC200 contributes to breast cancer pathogenesis. Cell Death Dis 7: e2262, 2016.

50. Yin Y, Zhong J, Li SW, Li JZ, Zhou M, Chen Y, Sang Y and Liu L: TRIM11, a direct target of miR-24-3p, promotes cell proliferation and inhibits apoptosis in colon cancer. Oncotarget 7: 86755-86765, 2016.

51. Cheng J, Roden CA, Pan W, Zhu S, Baccei A, Pan X, Jiang T, Kluger Y, Weissman SM, Guo S, et al: A molecular chipper technology for CRISPR sgRNA library generation and functional mapping of noncoding regions. Nat Commun 7: 11178, 2016.

52. Shechner DM, Hacisuleyman E, Younger ST and Rinn JL: Multiplexable, locus-specific targeting of long RNAs with CRISPR-Display. Nat Methods 12: 664-670, 2015.

53. Plummer RJ, Guo Y and Peng Y: A CRISPR reimagining: New twists and turns of CRISPR beyond the genome-engineering revolution. J Cell Biochem, 2017. https://doi.org/10.1002/jcb.26406.

54. Vojta A, Dobrinić P, Tadić V, Bočkor L, Korać P, Julg B, Klasić M and Zoldoš V: Repurposing the CRISPR-Cas9 system for targeted DNA methylation. Nucleic Acids Res 44: 5615-5628, 2016.

55. Amabile A, Migliara A, Capasso P, Biffi M, Cittaro D, Naldini L and Lombardo A: Inheritable silencing of endogenous genes by hit-and-run targeted epigenetic editing. Cell 167: 219-232, 2016.

56. Liu XS, Wu H, Ji X, Stelzer Y, Wu X, Czauderna S, Shu J, Dadon D, Young RA and Jaenisch R: Editing DNA methylation in the mammalian genome. Cell 167: 233-247, 2016.

57. Hilton IB, D'Ippolito AM, Vockley CM, Thakore PI, Crawford GE, Reddy TE and Gersbach CA: Epigenome editing by a CRISPR-Cas9-based acetyltransferase activates genes from promoters and enhancers. Nat Biotechnol 33: 510-517, 2015.

58. Pineda M, Moghadam F, Ebrahimkhani MR and Kiani S: Engineered CRISPR systems for next generation gene therapies. ACS Synth Biol 6: 1614-1626, 2017.

59. Beliveau BJ, Joyce EF, Apostolopoulos N, Yilmaz F, Fonseka CY, McCole RB, Chang Y, Li JB, Senaratne TN, Williams BR, et al: Versatile design and synthesis platform for visualizing genomes with oligopaint FISH probes. Proc Natl Acad Sci USA 109: 21301-21306, 2012.

60. Heun P, Laroche T, Shimada K, Furrer P and Gasser SM: Chromosome dynamics in the yeast interphase nucleus. Science 294: 2181-2186, 2001

61. Chen B, Gilbert LA, Cimini BA, Schnitzbauer J, Zhang W, Li GW, Park J, Blackburn EH, Weissman JS, Qi LS and Huang B: Dynamic imaging of genomic loci in living human cells by an optimized CRISPR/Cas system. Cell 155: 1479-1491, 2013.

62. Miyanari Y, Ziegler-Birling C and Torres-Padilla ME: Live visualization of chromatin dynamics with fluorescent TALEs. Nat Struct Mol Biol 20: 1321-1324, 2013.

63. Roukos V, Voss TC, Schmidt CK, Lee S, Wangsa D and Misteli T: Spatial dynamics of chromosome translocations in living cells. Science 341: 660-664, 2013.

64. van Steensel B and Dekker J: Genomics tools for unraveling chromosome architecture. Nat Biotechnol 28: 1089-1095, 2010.

65. Nelles DA, Fang MY, O'Connell MR, Xu JL, Markmiller SJ, Doudna JA and Yeo GW: Programmable RNA tracking in live cells with CRISPR/Cas9. Cell 165: 488-496, 2016.

66. Wang S, Su JH, Zhang F and Zhuang X: An RNA-aptamer-based two-color CRISPR labeling system. Sci Rep 6: 26857, 2016.

67. Jaitin DA, Weiner A, Yofe I, Lara-Astiaso D, Keren-Shaul H, David E, Salame TM, Tanay A, van Oudenaarden A and Amit I: Dissecting immune circuits by linking CRISPR-pooled screens with single-Cell RNA-Seq. Cell 167: 1883-1896, 2016.

68. Guernet A, Mungamuri SK, Cartier D, Sachidanandam R, Jayaprakash A, Adriouch S, Vezain M, Charbonnier F, Rohkin G, Coutant S, et al: CRISPR-barcoding for intratumor genetic heterogeneity modeling and functional analysis of oncogenic driver mutations. Mol Cell 63: 526-538, 2016.

This work is licensed under a Creative Commons

Attribution-NonCommercial-NoDerivatives 4.0 International (CC BY-NC-ND 4.0) License. 